\title{
Topical Issue on Opportunities for Lattice Gauge Theory in the Era of Exascale Computing
}

Published online: 14 November 2019 - C Società Italiana di Fisica / Springer-Verlag GmbH Germany, part of Springer Nature, 2019

This issue presents a set of seven white papers authored by the USQCD Collaboration spanning the diverse areas of nuclear and particle physics and computing in which Lattice Quantum Chromodynamics plays an important role. The white papers cover the topics:

1) Hadrons and nuclei.

2) Hot-dense Lattice QCD.

3) Opportunities for Lattice QCD in quark and lepton flavor physics.

4) Lattice QCD and neutrino-nucleus scattering.

5) The role of Lattice QCD in searches for violations of fundamental symmetries and signals for new physics.

6) Lattice gauge theory for physics beyond the Standard Model.

7) Status and future perspectives for lattice gauge theory calculations to the exascale and beyond.

There is some overlap among these topics and, in particular, the white paper on neutrino physics was written to highlight the important roles lattice QCD can play in neutrino physics, a field that spans both nuclear and particle physics. The white papers were written to support a renewal of the US DOE LQCD infrastructure project, which provides computational hardware for lattice QCD in the US. The scope defined for these white papers was to outline recent progress in particular by members of the USQCD Collaboration as well as to outline opportunities for the future. To this end, they are not intended to be comprehensive reviews of the subjects that they cover but represent a snapshot of the field. 